\title{
Ontology Design for Thailand Travel Industry
}

\author{
Sanya Khruahong, Xiaoying Kong, and Doan Hoang
}

\begin{abstract}
Tourism industry plays a crucial role in the economics growth in countries of South East Asia and hence the demand for rich local tourist information for visitors. In these countries, there are unique deciding impact factors to visitor journey such as constraints in culture, laws, and festivals. Such special information has not been available or implemented in general search engines so far. In this paper, we design an ontology for Thai travel industry to support an intelligent finding of these local events and their constraints using semantic web. The primary contribution of this paper is a new approach to design ontology for Thailand travel industry on both general information and specific dynamic local information using Domain Ontology Graph (DOG) and location based services. As a result, this ontology design will be applied to an intelligent searching for making decision of tourists. This ontology design will be used in semantic tourism applications in the future.
\end{abstract}

Index Terms-Location-based service, ontology, semantic tourism, semantic web, travel ontology.

\section{INTRODUCTION}

Recently, tourists of Southeast Asia have rapidly increased in this countries group. The ASEAN Economic Community (AEC) includes ten countries of Southeast Asia. In 2015, all countries of ASEAN will transformed into a region with free trade of goods, services, investment, and skilled labors and this fulfills the goal of regional economic integration [1]. Therefore, the tourism industry should be concentrated on developing in the areas of growth of the region.

Considering the unique culture activities and constraints in this region, foreign travelers have special needs to obtain the particular local and dynamic information during their travel. Although, countries of ASEAN have many similarities of tradition, but some particular information is different in the local setting. For example, some activities and information for Songkran festival in Thailand are different from Songkran festival in Cambodia, or the constraints of temple may be different in each suburb.

Therefore, special local travel information is necessary to assist travelers to plan their journey efficiently and cost effectively. Such information should include both general information and specific information of the region. General information consists of detail of attractive places, traffic report, transportation, or weather report, etc. Specific information consists of culture, laws, tradition, festivals and constraints that relate to the trip of tourists. Particularly, some festivals are related to religious calendar such as Buddhism, Islam, or Christianity. For example, many Thailand festivals

Manuscript received April 10, 2015; revised August 25, 2015.

The authors are with University of Technology Sydney, Faculty of Engineering and Information Technology, Sydney, Australia (email: Sanya.Khruahong-1@student.uts.edu.au, Xiaoying.Kong@uts.edu.au, Doan.Hoang@uts.edu.au). are related to Buddhism calendar and the activities often change to reflect the updated contents. Furthermore, some constraints are beneficial to their travel and decision making, for example, while tourists are visiting Thailand, they should abide by Thailand laws such as [2]:

"The sale of alcohol in Thailand is banned between 2pm and 5pm and again between midnight and 11 am seven days a week, and elections day, and religious holidays."

"The legal driving age in Thailand is 18 for car drivers and at least 15 years of age for motorbike drivers which are required to have an International Drivers 'License all time"

These rules could impact their journey-plan; therefore the tourists should be aware of and take these constraints into consideration before embarking on their activity. Normally, travellers search for travel information by using web based or smart phone based search applications such as Google, Yahoo and Baidu. However, current applications lack support for local constraints and specific tourist information needs of the region.

Furthermore, many applications have been designed using different technologies with contents from various sources. As a result, there is massive amount of information hidden in websites, which should be managed and mined for value-added actions. Understanding of computer in natural language is very difficult, therefore, if searching application system can be designed completely to automate the process, it will improve the chances of making the Semantic Web a reality [3].

The Semantic Web is an effort by the W3C (The World Wide Web Consortium) to enable integration and sharing of data across different applications and organizations which use knowledge represented in the W3Cs ontology web language (OWL) [4], [5]. It is a combination of different content, information applications, and systems across Internet [6]. Therefore, data management of semantic web should be done in efficient method. Ontology has been a part of the semantic web technique to serve as a dynamic database. In this paper, we design ontology in native information and general information for Thailand tourism industry. This ontology design includes using semantic database and Location-based Services (LBS) to efficiently support travellers' decision making. The ontology design flow is adapted using Knowledge Seeker. Domain ontology graphs (DOG) is used to implement dynamic database. This ontology will be applied in conjunction with location based services for dynamic filtering the local information in both general and specific information.

This paper is organized as follows. In Section II, we review the literature in ontology models such as top-level ontology, lexical ontology, and domain ontology, and location-based service. In Section III, we present an ontology design framework. Section IV specifies the ontology design for Thai travel industry. Section $\mathrm{V}$ concludes this research and 
discusses future research directions.

\section{RELATED WORKS}

In this section, we will review the related work on Thailand tourism and ontology design.

\section{A. Thailand Tourism}

Recently, tourism industry has been a major factor driving the growth of economic development in Southeast Asia. The number of international tourists has increased over the last twenty years. Thailand is an extremely attractive tourist destination [7]. It has vast cultural attractions, gorgeous beaches, amazing islands and national parks, providing tourists a rich variety of travel experiences. Statistics from Thailand Tourism Department show that 24.5 million tourists came to Thailand in 2013, from 22.3 million in 2012 and a target of 30 million visitors in 2015 [8]. Tourism competitiveness is an essential economic indicator. Therefore it is a major element of the economy of Southeast Asia and Thailand.

Currently travelers use many static methods for obtaining tourism information, such as brochures, guide books, and static website, but they are often outdated for proper journey planning. Techniques were developed and deployed for supporting travelling a decade ago. Especially, Thailand has made efforts in building internet tools for solving problems with tourism. These tools and information are mainly provided using Thai language not suitable for majority of travelers who only understand English. Furthermore, it is essential to expose general and specific, static and dynamic local information for tourists to plan their journey efficiently and cost-effectively.

Search engine is an important tool for travellers to search for information in Thailand. Google provides many services such as Google search, Google translate, and Google maps. Especially, Google maps offer street maps, street view, and satellite view, finding route and traffic status. However these Google tools do not provide travelers special local dynamic information such as culture, law and festival constraints. Therefore, there is a need to develop more effective dynamic location based searching applications for travellers. Ontology could be developed to serve as a dynamic sematic database to support the local information.

\section{B. Travel Ontology}

Ontology is a specification of a conceptualization and is a representation of a shared understanding of the crucial concepts in some domain of interest [9]. It becomes the semantic modeling technique. Ontology defines the term of specific domain, represents an area of knowledge and analyzes the contexts of the specific domain. It has three fundamental pieces requisite for a fully functioning semantic database: dictionary encoding, Resource Description Framework (RDFS) inference, and query processing. Ontologies modeling in computer systems have three models [10]: top level ontologies, lexical ontologies, and domain ontologies.

Domain ontology is developed under the concept formation in the specific domain of interest (Lim, 2011). Domain ontology is applied to specific domain from upper ontology. It is preferably built based on an available top-level ontology for mapping and integration between different domain ontology (Lim, 2011). Among domain ontology design approaches, the domain ontology graphs (DOG) produces high classification accuracy in a well-constructed ontology (Lim, 2011). DOG has two key components: the definition of the ontology graph and the ontology learning process (Liu et al. 2013, Liu et al. 2014). DOG methodology is a semi-automatic process which is suitable for our ontology design process that needs manual processing and automatic processing for considering both static and dynamic local tourism information.

In the past, web service was built to provide travel information services between peer-to-peer websites [11]. It was designed for semantically travel Web services. Web service is authorized from other web before using the services; it may suitable to some systems that the information is strictly analyzed without enough data to synthesis correct result.

Traditional relational database model [12] has been used for travel web services. For example, public transport relational databases stores information for travel bookings, payments, passengers, schedules, and timetables. However, the data and data structure in relational database are static without updating dynamic information semantically for tourists' special interests. Therefore semantic web is adapted to tourism for travel recommendation [13]. For example, Damljanovic and Devedžic presented ontology design for semantic web to manage massive data for travel recommendation. In addition to travel suggestion, retrieval of real-time travel information using ontology is another example in semantic web. For example, task model and task ontology presented by [14] are used to provide various intelligent tourist information. Travel recommendation system was developed by using Tourist Contents Service (TCS) and Task-Orient Menu Service (TMS) to provide real time information to travellers.

Among the travel ontology design approaches, some methods considered the linguistic knowledge with the context knowledge. TOCM (Tourism Ontology Construction Method) integrates the linguistic knowledge with the context knowledge using mathematical tools of Formal Concept Analysis (FCA). This method is applied for China tourism only [13]. Other methods include multi-stage ontology-based touristic recommender by analyzing user's profile of Facebook [14]. Using properties, relationships of travel ontology for the tourist suggestion more intelligent information by Chang et al. [15].

Among the current approaches, none of them has considered the design for local travel constraints such as culture, law and festival needs. Our research will fill in this gap and design such ontology for searching services in Thailand travel industry.

\section{Location-Based Services}

Location-based Services (LBS) is an important component of the mobile infrastructure, which it can supply to users with the crucial information depending on where users are current position [16]. It is a general class of computer program-level services used to include specific controls for location and time data as control features in computer programs. They are developed with many tools which they can support to user traveling.

A number of recent articles analyses how applications are 
designed by using LBS [15], [17]. While, Seiji Yokoji report how LBS apply to searching documents on the internet. While Martin Espetera and Raubal explain that LBS is applied on mobile to finding the restaurant in the city by using simulating for looking to optimal results of searching. These articles express a similar need to include a location-based search engine in their applications. The authors agree that it is an important "point to locate" feature for searching. According to Yokoji et al. location-based search engine was developed for searching algorithm on distance between a user-specified location and locations that are described in web documents.

Espetera and Raubal explain that the prototype is used for simulating a location-based decision process aimed at finding an optimal restaurant for a user group in an urban. However, the paper focuses on search engine for restaurant only. LBS prototype is applied with methods of multi-criteria decision-making, time geography and defining of similarity measurement.

In contrast, Yokoji et al. used these latitude-longitude pairs to retrieve documents. The scheme consists of three modules: a module for collecting documents from the Internet, a module for analyzing of strings from web documents for finding latitude-longitude to the original document, and a retrieval module. In brief, LBS can be used with smart mobile devices to search and extract relevant action.

Recently, the government of New South Wales has adopted the NSW Location Intelligence (LI) strategy with the aim of maximizing the value of location intelligence in decision making, planning and service delivery for the benefit of the NSW Government, industry and communities [18]. It will be applied in smart city environments deploying location based information such as an address, a postcode, and a building. The strategy is being adapted with types of business information such as financial data, service delivery data, and client information which able to make decision for users.

\section{AN ONTOLOGY DESIGN FRAMEWORK}

In this section, we present the adopted framework for ontology design. This framework will be used to generate the domain ontology graph (DOG) and manage the document classification. The ontology graph is created as a graphical structure within the framework of the Knowledge Seeker system [10] and its representation is used for intelligent information application development.

\section{A. The Framework for Ontology Modeling}

Knowledge Seeker is a framework for ontology design [10]. It defines and implements components of four main modules.

Ontology Modeling (the ontology structure): The ontology modeling module is used to represent the ontology data in the Knowledge Seeker system in which the knowledge is represented as ontology graph.

Ontology Learning (the learning algorithm): The ontology learning module defines the method of conceptualizing a domain of knowledge.

Ontology Generation (the format): The ontology generation module uses a text corpus to generate domain ontology in the ontology graph form and graphical format.

Ontology Query (the operations): The ontology querying module is crucial module that uses Knowledge Seeker system to develop intelligent applications such as text classification systems and text searching systems.

\section{B. Methodology on Ontology Learning}

The content-based ontology learning process is applied to our design to enrich the ontology and its validation. The approach of this ontology design is based on the ontology learning process and the knowledge ontology modeling [10], [19] with four steps as shown in Figure 1.

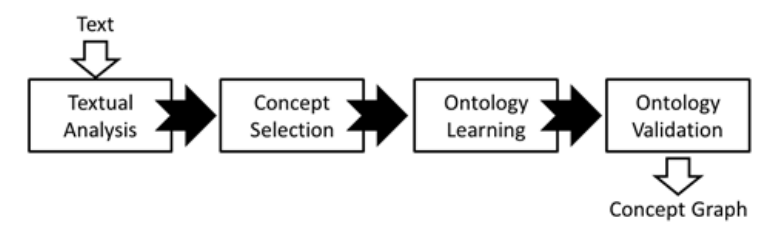

Fig. 1. Contest-based ontology learning process.

Textual analysis process: Web channels documents are applied as the initial term list to extract a list of candidate terms (CT) which are meaningful words in human perspective.

Concept selection process: this process aims to select a concept set that is lerated related to web channels. A cConcept (C) is one or more candidate terms groupeds together that relates to other knowledge unit. Compared to the excepted frequency $E_{i, j}$ where $i \in\{t, \neg t\}$ and $j \in\{c, \neg c\} . E_{i, j}$ is defined as:

$$
E_{i, j}=\frac{\sum_{a \in\{t, \neg t\}} O_{a, j} \sum_{b \in\{c, \neg c\}} O_{i, b}}{N}
$$

The statistics of Chi-square for term $t$ and class $\mathrm{c}$ is defined as:

$$
X_{t, c}^{2}=\sum_{i \in\{t, \neg t\}} \sum_{j \in\{c, \neg c\}} \frac{\left(O_{i, j}-E_{i, j}\right)^{2}}{E_{i, j}}
$$

Ontology learning process: the concept relation (CR) is measured between every concepts in the class. CR defines how two concepts relate to each other. Equation is changed as to measure for term $t_{a}$ and term $t_{b}$.

$$
X_{t_{a}, t_{b}}^{2}=\sum_{i \in\left\{t_{a}, \neg t_{a}\right\}} \sum_{j \in\left\{t_{b}, \neg t_{b}\right\}} \frac{\left(o_{i, j}-E_{i, j}\right)^{2}}{E_{i, j}}
$$

Ontology validation process: this process aims to determine the fitness of an Ontology Graph in representing a class of Web channel. Each document is represent $d$ by a term-frequency vector $T F=\left\langle t f_{1}, t f_{2}, \ldots, t f_{n}\right\rangle$, and score vector is calculated for document $\mathrm{d}$ for every term $t_{i}$. The score function $S_{i}$ for a term $t_{i}$ is defined as:

$$
S_{t_{i}}=\left\{\begin{array}{l}
X_{t_{i, c}}^{2} \text { if } t_{i} \text { exist in } d \text { else } \\
0
\end{array}\right.
$$

\section{TRAVEl ONTOLOGY DESIGN}

By adopting the design approach in Fig. 1, we will demonstrate the travel ontology design in this section.

As an initial step, this paper focuses only on the ontology 
design for travel, both general static information and local specific and dynamic information. We describe the conversion from the original source of the text to the proposed ontology modeling, as shown in Fig. 2. After ontology process, a dynamical database is built to provide the fundamental information of tourism system that will be used in applications for both web and mobile phones such as recommendation system, decision support system, question answering system, or semantic search system. Moreover, LBS will be adapted to improve efficient of system by dynamically filtering particular data of classes.

This research will design the specific data of tourism in Thailand based on the collected static information and dynamic information. Target of the research is the travellers who use the internet for searching. This research will identify the context and extract domain by using reliable sources from Thailand, and will use location-based services to support general and specific information. In addition, we adopt 3 modules from the Knowledge Seeker system.

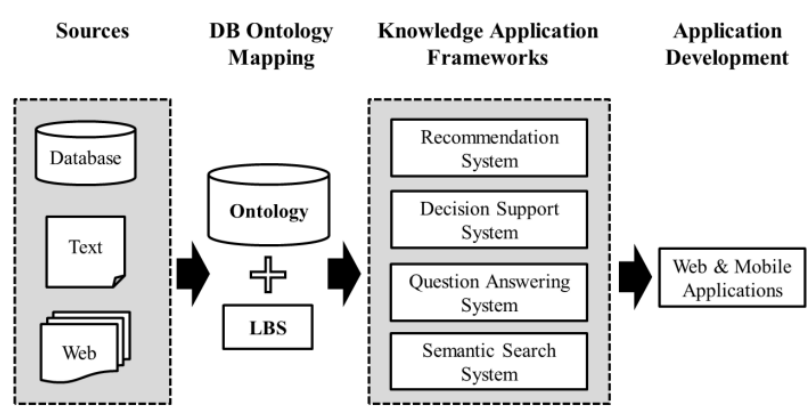

Fig. 2. Ontology design process.

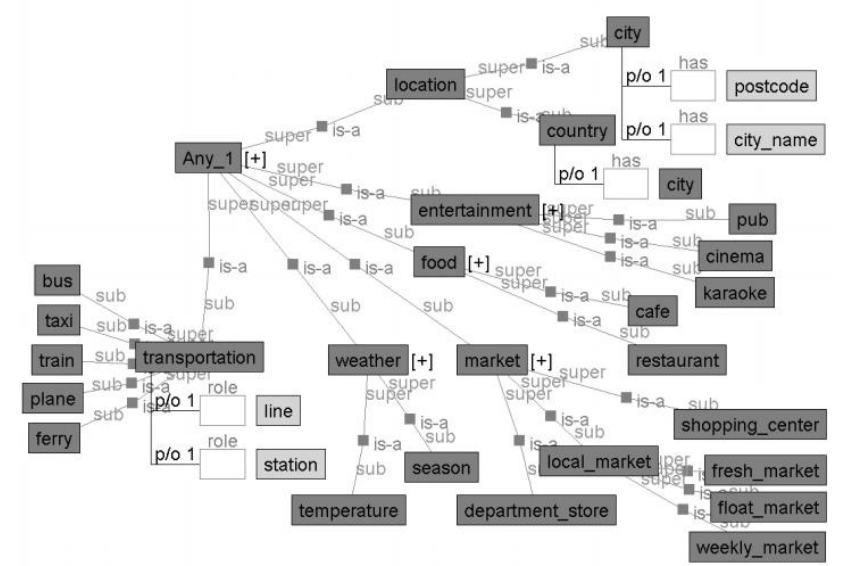

Fig. 3. Graphical ontology on general information.

Ontology for Thailand travel industry is designed into two types: travel domain ontology and travel task ontology. The travel domain ontology contains the static information which changes slowly over time such as information about attractive place, hotel, restaurants information etc. the travel task ontology includes the dynamic information that rapidly changes. Sometime data in the travel task ontology may change on a daily basis such as weather report, traffic report etc. In our design, both static and dynamic information are adapted to using general information and particular information in the local context.

Fig. 3 shows a part of design diagram of the travel ontology for general information. Our ontology has 2 main nodes that Any_1 is general information class as Fig. 3 and Any_2 is specific information class as Fig. 4. For example, the Any_1 class represents a set of general information on tourism ontology such as entertainment, food, market, transportation or weather. Each class has its Any_1; the relation with location class is defined as show location. Therefore, actions and properties will represent on current position by location class which leads to dynamic values. The location class is the area name which is searched by using GPS or location based service technology. Therefore, it will show the information of traveller's current position such as suburb name, city, province or country.

Table I shows some classes of Any_1 for general information ontology. For instance, basic local information, entertainment, food, transportation, or weather. They have many sub-classes which related to main general information.

TABLE I: EXAMPLE OF ELEMENT DESCRIPTION OF GENERAL INFORMATION The elements of general information

Basic Local Information (0)

General information about Thailand, Historicity, Attractive places, Tradition

\begin{tabular}{l}
\hline Entertainment (1) \\
Cinema, Karaoke, Pub \\
\hline Food (2) \\
Thai restaurant, Café, Promotion \\
\hline Transportation(3) \\
Route, Line, Traffic report \\
\hline Weather (4) \\
Temperature report, Season
\end{tabular}

Fig. 4 shows a part of design the travel ontology on specific information. Similar to Fig. 3, Any_2 is shown on particular information classes. It is consisted of things that link to constraints and particular data. These things are the specification of classes that link to particular information of travelling such as culture, laws, festival or constraints. However, Any_2 is related to Any_1 with a location class. The constraints of Any_2 will be supported to main point of travel information on Any_1. It is expanded some local information need and detail many sub-classes which is very advantageous for tourists.

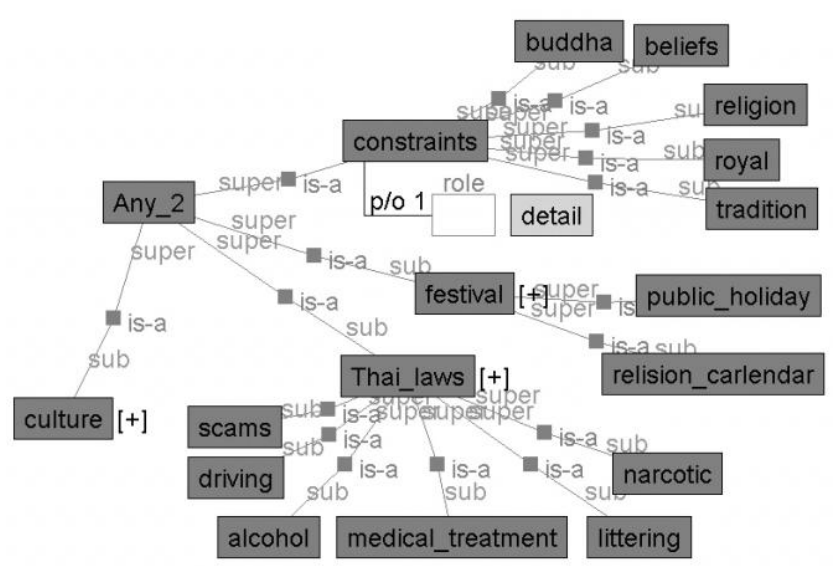

Fig. 4. Graphical ontology on specific information.

Table II shows some classes of Any_2 for specific information ontology. It is related to the constraints that tourists should understand. 
TABLE II: EXAMPLE OF ELEMENT DESCRIPTION OF SPECIFIC INFORMATION The elements of specific information

Basic Specific Information (0)

Specific information about Thailand, Constraints

Culture (1)

Cultural Constraints, Buddha, Royal, Beliefs, Religion,

Festivals (2)

Festival related to Religion calendar, Buddha, Religion

Thai Laws (3)

Driving Law, Alcohol Law, Vat Refund, Medical Treatment, Littering, Scams, Narcotic, Arrested in Thailand

For implementation, after design process, we will developit to Ontology Web Language (OWL) for next process. OWL is the markup language used in Semantic Web architectures.

Finally, to validate the quality of this ontology design, we evaluate the error rate by the information retrieval model which aims to calculate the retrieval accuracy, measurement in term of F-measure from Recall and Precision for evaluation of searching [10].

Precision, measures the percentage of accuracy of the retrieval model. It is defined by:

$$
\text { Precision }=\frac{T P}{T P+F P}
$$

Recall measures the percentage of correctly retrieved documents to all the documents. It is defined by:

$$
\text { Recall }=\frac{T P}{T P+F N}
$$

F-Measure measures the average of precision and recall. It is defined by:

$$
F-\text { Measure }=\frac{2 \times \text { Precision } \times \text { Recall }}{\text { Precision }+ \text { Recall }}
$$

See the design is evaluated through the tabulation of the retrieval accuracy from the actual result, as shown in Table III.

\begin{tabular}{lcc}
\multicolumn{2}{c}{ TABLE III: THE RETRIEVAL RESULT TABLE } \\
\hline \multirow{2}{*}{ Retrieved } & Relevant & Irrelevant \\
Not Retrieved & $F P$ & $F N$ \\
& & $T N$ \\
\hline
\end{tabular}

TP (True Positive): the number of relevant documents, retrieved as relevant.

FP (False Positive): the number of relevant documents, not retrieved as relevant.

FN (False Negative): the number of not relevant documents, retrieved as not relevant.

TN (True Negative: the number of not relevant documents, not retrieved as relevant.

\section{CONCLUSIONS AND FutURE RESEARCH DiRECTIONS}

In this paper, we designed ontology for Thailand travel industry. This ontology considered both static and dynamic special local information in culture, law and festival information. We have designed this travel ontology using DOG and LBS. DOG is created from a large classified text in the domain ontology learning process. The ontology graph consists of different levels of conceptual units and terms that are associated together by different kinds of relations. Moreover, LBS is used to extract the crucial location-specific data on ontology classes with area name obtained from smart phone's GPS.

This ontology design can be adapted to specific travel industries for developing many classes of intelligent applications, both web applications and smart phone applications.

This is our first step for designing the location based services for Thailand travel industry. In the future studies, we will research on more specific information of Thailand which may be specially impact to travel ontology design such as the political issues and the economic issues.

\section{REFERENCES}

[1] ASEAN Secretariat. ASEAN Economic Community Blueprint. (2008) [Online]. Available: http://www.asean.org/archive/5187-10.pdf

[2] J. Patin. Thailand Tourist Information A Guide to Laws in Thailand. (2011). [Online]. Available: http://www.thailawforum.com/tourst-guide-laws-Thailand.html

[3] Y. Tijerino et al., "Towards ontology generation from tables," World Wide Web, vol. 8, no. 3, pp. 261-285, 2005.

[4] T. Padiya, M. Bhise, and S. Chaudhary, "Semantic web data partitioning," Advancing Information Management through Semantic Web Concepts and Ontologies, 2013, pp. 154-165.

[5] R. Fikes, P. Hayes, and I. Horrocks, "OWL-QL-a language for deductive query answering on the Semantic Web," Web Semant, vol. 2 , no. 1, pp. 19-29, 2004.

[6] A. I. Walisadeera, G. N. Wikramanayake, and A. Ginige, "An ontological approach to meet information needs of farmers in Sri Lanka," in Proc. International Conference on 13th Computational Science and Its Applications 2013, pp. 228-240.

[7] W. Greene, Growth Forecasts for Southeast Asian Travel and Tourism (2012 - 2015). (2013). [Online]. Available: http://www.tigermine.com/2013/01/09/growth-forecasts-southeast-asi a-travel-tourism/

[8] Business-in-Asia.com. Time to Improve Thai Tourism. (2013) [Online]. Available: http://www.business-in-asia.com/thailand/thailand_tourism_2013.htm 1

[9] S. H. Othman, "Supporting domain ontology through a metamodel: A disaster management case study," Ontology-Based Applications for Enterprise Systems and Knowledge Management, 2013, pp. 191-209.

[10] E. H. Y. Lim et al., "Knowledge seeker-ontology modelling for information search and management: a compendium," Intelligent Systems Reference Library, vol. 8, 2011.

[11] A. Dogac et al., "Semantically enriched web services for the travel industry," SIGMOD Rec., vol. 33, no. 3, pp. 21-27, 2004.

[12] D. Damljanovic and V. Devedžic, "Applying semantic web to e-tourism," The Semantic Web for Knowledge and Data Management, 2009, pp. 243-265.

[13] T. Suqin and C. Zixing, "Tourism domain ontology construction from the unstructured text documents," presented at 9th IEEE International Conference on Cognitive Informatics (ICCI), 2010.

[14] K. Alonso et al., "Ontology-based tourism for all recommender and information retrieval system for Interactive Community Displays," presented at 8th International Conference on Information Science and Digital Content Technology (ICIDT), 2012.

[15] M. Espeter and M. Raubal, "Location-based decision support for user groups," Journal of Location Based Services, vol. 3, no. 3, pp. 165-187, 2009.

[16] T. Orehovacki, Z. Stapic, and G. Bubas, "Mobile location based service for location and presentation of cultural heritage objects and web 2.0 technologies," vol. 42, 2009.

[17] Y. Seiji, T. Katsumi, and M. Nobuyuki, "Kokono search: A location based search engine," presented at International Conference on Tenth International World Wide Web Conference, 2001, Hong Kong, China. 
[18] NSW_Government. Priorities: NSW Location Intelligence Strategy. (2014). [Online]. Available: http://www.finance.nsw.gov.au/ict/priorities/managing-information-b etter-services/nsw-location-intelligence-strategy

[19] T. Guoxin, H. Tinglei, and Z. Zheng, "A knowledge modeling framework for intangible cultural heritage based on ontology," presented at Second International Symposium on Knowledge Acquisition and Modeling, 2009.

Sanya Khruahong received the BSc degree in computer science from Pibulsongkram Rajabhat University, Thailand, in 1997, and the MSc in information technology from King Mongkut's University of Technology North Bangkok in 2003. Recently, he is studying in Faculty of Engineering and Information Technology at University of Technology, Sydney (UTS). His research interests include search engine, web technologies, ontology, web search, mobile web search, and localization.
Xiaoying Kong is a senior lecturer in the School of Computing and Communications at University of Technology, Sydney (UTS). She is a member of Centre for Real-Time Information Networks. She has broad interests in control engineering and software engineering.

Doan B. Hoang received the BE degree from the University of Western Australia, and the ME and PhD degrees from the University of Newcastle, Australia. He is a professor in the School of Computing and Communications, Faculty of Engineering and Information Technology, University of Technology, Sydney (UTS). He is the director of iNEXT and the director of the Advanced Research in Networking (ARN) Laboratory. 